\title{
Implicaciones hepáticas en la pandemia por COVID-19
}

\section{Liver implications during the COVID-19 Pandemic}

\author{
Juanita León Gómez, ${ }^{1}$ Andrés José Gómez Aldana, ${ }^{2}$ Mónica Lorena Tapias Mantilla. ${ }^{3 *}$
}

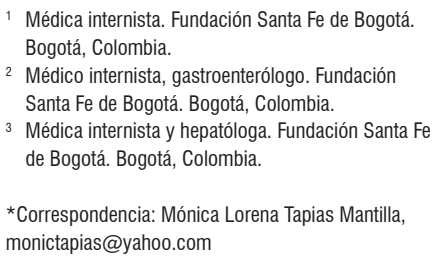

*Correspondencia: Mónica Lorena Tapias Mantilla, monictapias@yahoo.com

Fecha recibido: $\quad 04 / 04 / 20$ Fecha aceptado: 04/17/20

\begin{abstract}
Resumen
La infección generada por el coronavirus, denominado SARS-CoV-2, llamada coronavirus disease 2019 (COVID-19), surgió en China a finales de diciembre de 2019. Actualmente ha sido categorizada como una pandemia por la Organización Mundial de la Salud (OMS). Se han documentado alteraciones de pruebas hepáticas, sin embargo, los estudios se han enfocado en los efectos cardíacos, pulmonares y renales de esta infección. La alteración de pruebas hepáticas en el contexto de COVID-19 puede ser consecuencia de hepatitis viral, toxicidad farmacológica, inflamación o choque. También se considera como un marcador de pronóstico y gravedad de la enfermedad. El impacto de la infección por SARS-CoV-2 en pacientes con enfermedad hepática preexistente o receptores de trasplante hepático no es claro, y se plantean distintas hipótesis sobre mayor o menor riesgo de enfermedad grave y de descompensación de la enfermedad de base.
\end{abstract}

Palabras clave

SARS-CoV, SARS-CoV-2, MERS-CoV, hígado, trasplante de hígado, cirrosis, inmunosupresión.

\begin{abstract}
The infection generated by the novel coronavirus SARS-CoV-2, named Coronavirus Disease 2019 (COVID-19) emerged late December of 2019 in China. It is currently categorized as a pandemic by the World Health Organization. Studies have focused on cardiac, pulmonary, and renal effects of this infection, but liver test abnormalities have also been documented. This alteration may be a consequence of viral hepatitis, pharmacological toxicity, inflammation, or shock. It is also considered a marker of prognosis and severity of the disease. The impact of SARS-CoV-2 infection in patients with pre-existing liver disease or liver transplant recipients is unclear, and different hypotheses exist regarding the higher or lower risk of severe disease and decompensation of the underlying disease.

Keywords

SARS-CoV, SARS-CoV-2, MERS-CoV, liver, liver transplant, cirrhosis, immunosuppression.
\end{abstract}

\section{INTRODUCCIÓN}

La infección generada por el coronavirus, denominado SARS-CoV-2, llamada coronavirus disease 2019 (COVID19), surgió en China a finales de diciembre de 2019. Actualmente ha sido categorizada como una pandemia por la Organización Mundial de la Salud (OMS) (1). Hasta el
16 de abril se han documentado 2088425 casos de personas infectadas a nivel mundial (2). En Colombia, hasta la misma fecha, se han registrado 3105 casos de personas contagiadas y 131 fallecidos por esta enfermedad (3).

El SARS-CoV-2 es un coronavirus que hace parte del grupo de 7 especies de coronavirus que pueden generar infección en humanos, y comparte información genética 
con otros virus altamente patogénicos como el SARS-CoV y el MERS-CoV. Los tres virus generan infección respiratoria; en el caso de las infecciones por SARS-CoV y MERS$\mathrm{CoV}$ se ha documentado previamente daño hepático asociado con la infección $(4,5)$. Hasta el momento no se tiene mucho conocimiento sobre el impacto de la infección por SARS-CoV-2 en pacientes con enfermedad hepática establecida o en pacientes receptores de trasplante hepático.

El objetivo de esta revisión es resumir la información disponible hasta la fecha sobre el compromiso hepático en la infección por SARS-CoV-2 en pacientes con y sin enfermedad hepática preexistente, y en pacientes receptores de trasplante hepático.

\section{DAÑO HEPÁTICO}

Durante la epidemia de síndrome respiratorio agudo severo (SARS, por sus siglas en inglés) causado por el virus SARS-CoV en China en el 2002, se documentó que algunos pacientes presentaban una elevación leve a moderada de las transaminasas (6). En su momento se planteó que el daño hepático podía generarse por lesión viral directa, teniendo en cuenta que el virus utiliza la enzima convertidora de angiotensina 2 (ECA2) como su receptor y que esta abunda en el endotelio a nivel hepático y en la vía biliar $(7,8)$. Otros estudios demostraron que la proteína viral específica 7a tiene la capacidad de inducir apoptosis en varias líneas celulares, incluido en el hígado (9). Sumado a estos hallazgos, dos estudios documentaron la presencia de SARS-CoV en el tejido hepático (10). Mediante una biopsia hepática post mortem se han podido identificar cambios, como la esteatosis microvacuolar con actividad portal y lobular leve, que pueden ser causados por una lesión viral o daño inducido por medicamentos (6).

De manera similar, se ha demostrado que los pacientes con el síndrome respiratorio de Medio Oriente (MERS, por sus siglas en inglés), causado por el virus MERS-CoV, presentan una elevación de las transaminasas y de la bilirrubina, y un descenso de la albúmina sérica, lo cual se considera como un marcador de gravedad de la enfermedad (11-13). A diferencia del SARS-CoV y del SARS-CoV-2, el MERS-CoV utiliza la dipeptidil peptidasa 4 (DPP4) y su receptor para infectar a las células; se sabe que el hígado tiene alta expresión de esta enzima, y que este mecanismo podría favorecer el daño hepático en esta enfermedad (6). En la infección por SARS-CoV y MERS-CoV se ha estudiado una respuesta proinflamatoria con la elevación de interleucinas y otros reactantes de fase aguda, por lo que otra hipótesis plantea que el daño hepático también esté mediado. por esa respuesta inflamatoria (6).

El SARS-CoV-2 es un agente infeccioso identificado inicialmente en diciembre de 2019 en China. Los datos disponibles en ese país muestran que entre el $14 \%-53 \%$ de los pacientes con COVID-19 tienen una elevación de las transaminasas y que esa elevación es más frecuente en pacientes con enfermedad grave (7). En varias series de casos de pacientes con COVID-19 en China se reportó una elevación de la alanina aminotransferasa (ALT) y de la aspartato aminotransferasa (AST) en el 21,7 \% - $28 \%$ y el 29,8 \%-35 \% de los casos, respectivamente, con solo un paciente con una elevación alta de las transaminasas $(14,15)$. En un estudio de predictores de mortalidad en COVID-19 se identificó que la ALT elevada, la hipoalbuminemia y la trombocitopenia son predictores de mal pronóstico en el análisis univariado; sin embargo, en el análisis multivariado ningún parámetro mostró una correlación fuerte con desenlaces adversos (16). En otro estudio identificaron que los índices de disfunción orgánica, incluida la elevación de la AST, forman parte de los factores predictores del síndrome de dificultad respiratoria aguda (SDRA) (15). En uno de los pocos estudios en los que registraron el número de pacientes con enfermedad hepática crónica, no se identificó una asociación entre este antecedente y el pronóstico en COVID-19, aunque solo identificaron 4 pacientes de 138 individuos infectados (17). Teniendo en cuenta que pocos estudios han incluido la enfermedad hepática crónica como un antecedente relevante, es difícil saber si estas alteraciones en las pruebas analíticas son indicadores de enfermedad hepática preexistente o de hepatitis viral, o únicamente indicadores de gravedad de la enfermedad.

Es importante tener en cuenta que además del posible daño hepático causado por la infección viral y la respuesta inflamatoria asociada, muchos pacientes reciben medicamentos hepatotóxicos que pueden generar una alteración de las pruebas hepáticas $(6,11,13-16)$. Algunos de los medicamentos que se usan frecuentemente son la cloroquina e hidroxicloroquina, azitromicina, ribavirina, lopinavir más ritonavir y esteroides sistémicos $(18,19)$. Todos estos medicamentos se metabolizan por vía hepática y tienen un potencial de toxicidad que puede manifestarse con una alteración de las pruebas hepáticas con un patrón necroinflamatorio o colestásico (Figura 1).

\section{ENFERMEDAD HEPÁTICA PREEXISTENTE}

Los pacientes con enfermedad hepática crónica y cirrosis tienen pobre función inmune, por lo que se ha planteado que puedan tener peores desenlaces cuando presentan infección por SARS, SARS-CoV-2 y MERS-CoV (20-22). En la actualidad, solamente se cuenta con un cálculo de incidencia de COVID-19 en pacientes con cirrosis descompensada con un estudio que reporta 17 casos documentados en una cohorte de 101 pacientes con cirrosis descompensada en Wuhan (22). Hasta ahora no se han reportado 


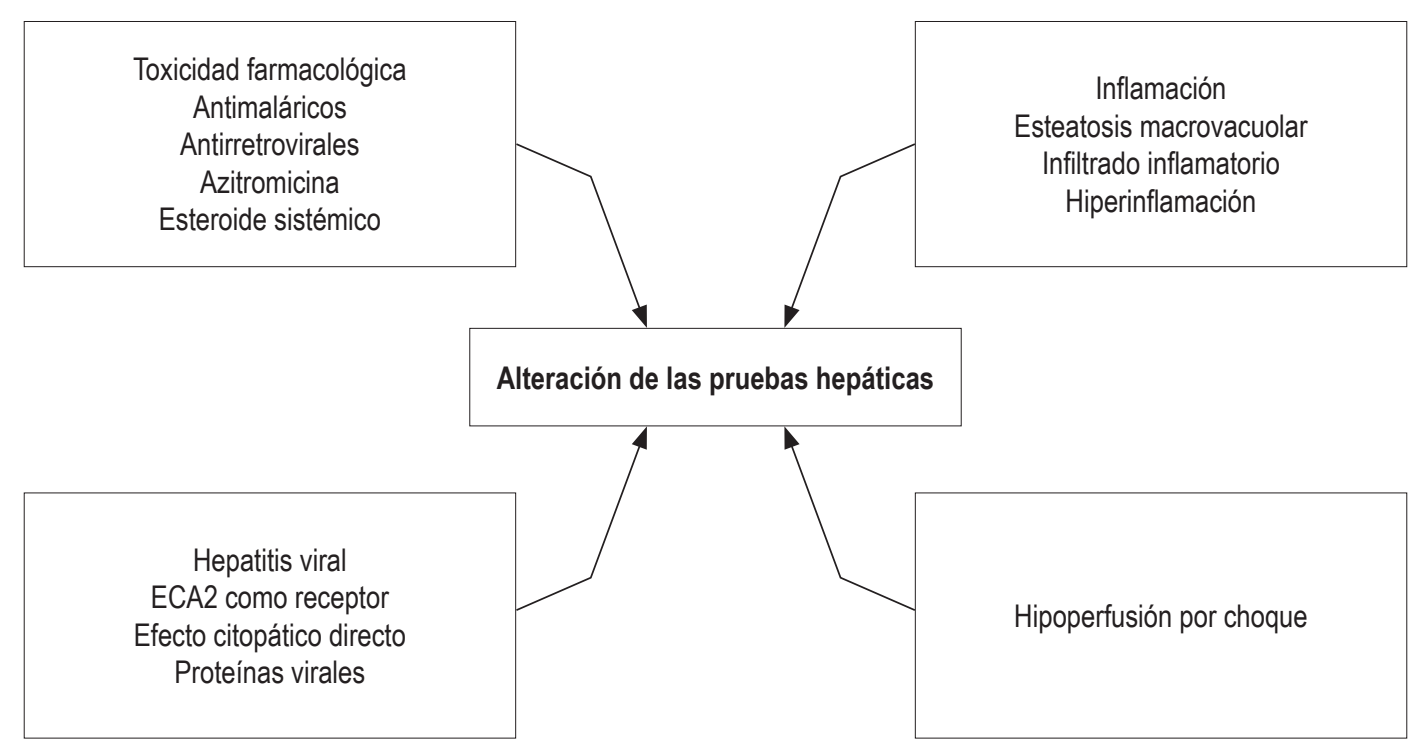

Figura 1. Alteración de pruebas hepáticas en COVID-19. La elevación de transaminasas y de bilirrubina, la hipoalbuminemia, la trombocitopenia y la prolongación de tiempos de coagulación son anormalidades que tienen distintas explicaciones fisiopatológicas y que tienen relación con la infección viral directamente, con la respuesta inmune del huésped y con otros atenuantes como el uso de fármacos hepatotóxicos.

casos de falla hepática o falla hepática aguda sobre crónica en pacientes con cirrosis con infección por COVID-19.

En la crisis actual, los mecanismos fisiopatológicos de la infección por SARS-CoV-2 pueden tener implicaciones especiales en los pacientes con enfermedad hepática previa, por ejemplo: lesión de colangiocitos y agravamiento de la colestasis en la colangitis biliar primaria o reactivación de la replicación viral en pacientes con hepatitis B en fase de tolerancia inmunológica, o con supresión de la replicación viral con entecavir $(13,21)$. En pacientes con infección por hepatitis virales crónicas (Hepatitis B y C) o por hepatitis autoinmune es posible que la linfopenia, el descenso de linfocitos $\mathrm{T} \mathrm{CD} 4^{+}$y el aumento marcado de citocinas durante la infección por SARS-CoV-2 puedan asociarse con un aumento de la replicación viral o un aumento de la actividad inflamatoria local debido a una mayor actividad autoinmune $(6,13,22)$. Existen otras hipótesis que plantean que, en pacientes inmunosuprimidos, la activación de macrófagos y el síndrome de hiperinflamación con tormenta de citocinas no son frecuentes, y que la inmunosupresión o la disfunción inmune podrían ser un factor protector (23).

Los pacientes con hígado graso o esteatohepatitis no alcohólica usualmente presentan comorbilidades significativas como diabetes mellitus, hipertensión arterial, obesidad y síndrome metabólico, que conllevan un mayor riesgo para las complicaciones por COVID-19. Por esta razón, es particularmente importante extremar medidas de prevención de contagio en esta población (13-16).
En un estudio realizado con 111 pacientes con cirrosis descompensada, que incluyó 29 pacientes en el 2020, se emitieron recomendaciones a pacientes sobre cómo evitar visitas y áreas concurridas, usar tapabocas, mantener ventanas abiertas y áreas bien ventiladas, el uso de un inhibidor de la bomba de protones por 2 meses después de ligadura endoscópica de várices esofágicas y la extensión de los intervalos de consulta para minimizar la exposición y el riesgo de contagio (24). Siguiendo estas recomendaciones, ningún paciente presentó síntomas sugestivos de infección por SARS-CoV-2. La Sociedad Europea de Enfermedad Hepática sugiere que en los pacientes con enfermedad hepática descompensada se realicen controles por telemedicina, que la toma de pruebas analíticas se haga en casa o en centros cercanos al paciente, que se mantengan los mismos cuidados médicos y farmacológicos sin modificaciones, que se reduzca al mínimo la evaluación pretrasplante hepático intrahospitalario, y que se incluya en lista de espera para trasplante hepático únicamente a pacientes gravemente enfermos (25). También se recomienda tomar prueba para SARS-CoV-2 en pacientes con falla hepática aguda sobre crónica o que presenten una descompensación aguda de la cirrosis (25).

La mayoría de los medicamentos que se usan para el tratamiento de COVID-19 tienen metabolismo hepático (13). No hay información sobre la incidencia de daño hepático por medicamentos específicamente en esta población, pero es importante tener en cuenta un posible aumento 
del riesgo de toxicidad por antimaláricos, antivirales y antibióticos en pacientes con enfermedad hepática subyacente (18). En los pacientes con cirrosis no hay información suficiente que indique si COVID-19 implica un mayor riesgo para la falla hepática aguda sobre crónica, la descompensación o las complicaciones relacionadas con la cirrosis.

La Sociedad Europea de Enfermedad Hepática sugiere que en los pacientes con enfermedad hepática compensada se utilicen alternativas como la telemedicina y la toma de pruebas analíticas en casa o en centros cercanos al paciente para minimizar la exposición, o que se pospongan los controles médicos; asimismo, no se recomienda modificar los esquemas de inmunosupresión en la hepatitis autoinmune para reducir el riesgo de infección por SARS-CoV-2 o complicaciones asociadas (25). Para los pacientes con carcinoma hepatocelular es importante continuar con los tratamientos locales y/o sistémicos en la medida que sea posible, teniendo en cuenta que es una enfermedad tiempo-sensible y que la progresión del tamaño tumoral puede ser una contraindicación para el trasplante en un plazo cercano (25).

En cuanto al tratamiento farmacológico en pacientes con COVID-19, la Asociación Americana de Estudio de Enfermedades Hepáticas recomienda considerar la reduc- ción de la dosis de esteroide sistémico manteniendo una dosis mínima de $10 \mathrm{mg} /$ día de prednisona o equivalentes para evitar la insuficiencia adrenal, y considerar la reducción de antimetabolitos (micofenolato y azatioprina) e inhibidores de calcineurina (tacrolimus y ciclosporina) únicamente en caso de linfopenia, fiebre o mayor progresión de la neumonía (26). La alteración de las pruebas hepáticas rara vez constituye una contraindicación para el uso de antivirales, antimaláricos o antibióticos; el uso de acetaminofén debe restringirse a máximo 2-3 g/día en pacientes con cirrosis; y el uso de antiinflamatorios no esteroideos (AINE) está contraindicado en pacientes con cirrosis e hipertensión portal (25). Es importante que los esquemas de vacunación se mantengan al día, según la edad y la comorbilidad del paciente (Figura 2) $(25,26)$.

\section{RECEPTORES DE TRASPLANTE HEPÁTICO}

Los receptores de trasplante hepático adquieren una alteración en la función inmune humoral y celular por la inmunosupresión farmacológica, y tienen un mayor riesgo de infección grave por agentes virales (24). Parte de la fisiopatología de COVID-19 incluye una respuesta inmunoló-

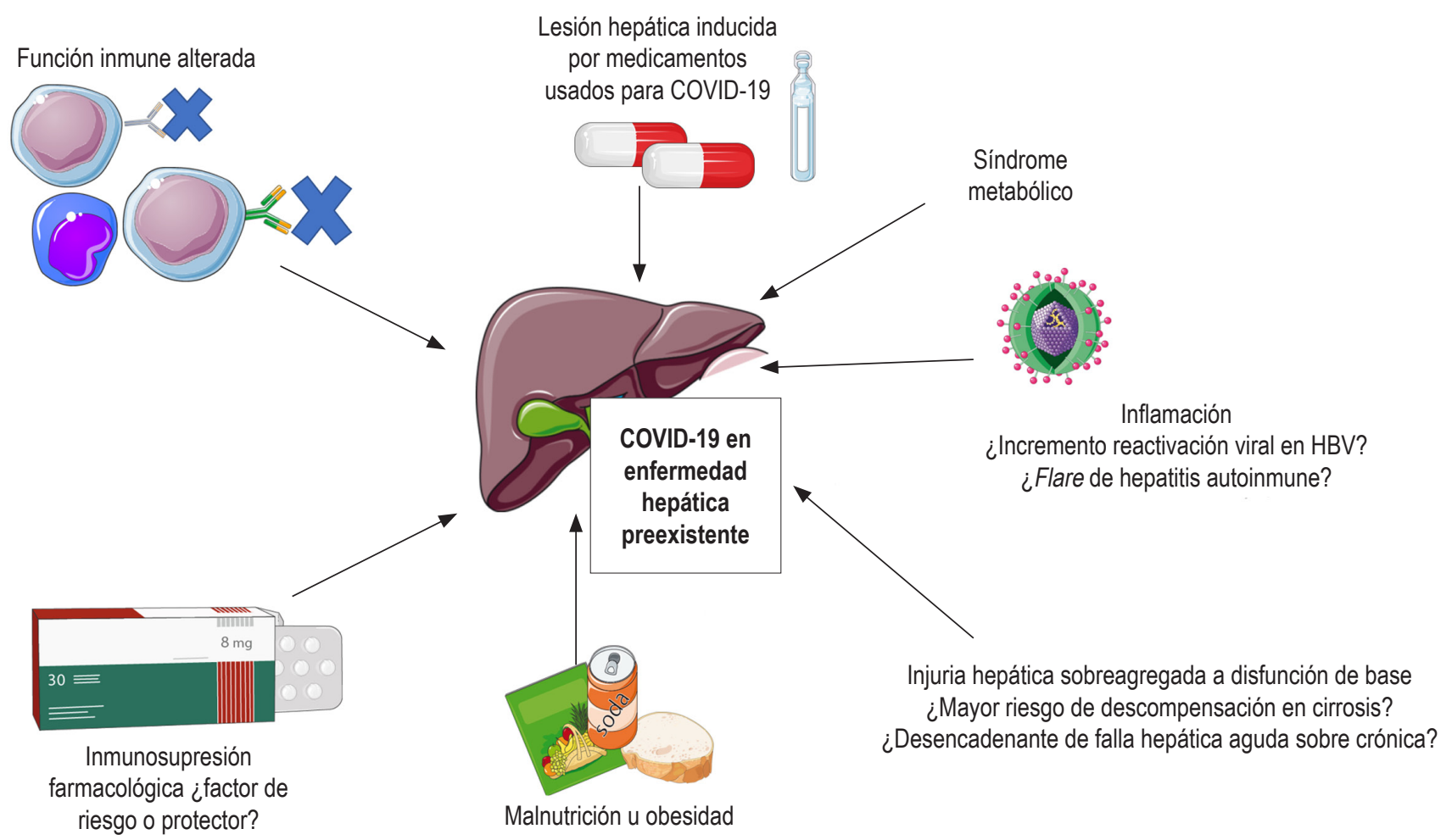

Figura 2. COVID-19 en enfermedad hepática preexistente. En este escenario se generan un gran número de interacciones complejas entre condiciones de base del paciente que pueden ser considerados factores de riesgo o factores protectores. 
gica aumentada que desencadena una cascada desregulada y excesiva (27). Existe muy poca información reportada en la literatura sobre los desenlaces de la infección por SARS-CoV, MERS-CoV y SARS-CoV-2 en receptores de trasplante hepático. Durante el brote de SARS en el 2002 se documentaron en la literatura algunos casos de pacientes adultos trasplantados que tuvieron neumonía grave, SDRA o un desenlace fatal, los cuales ocurrieron en presencia de otros factores de riesgo (28). No se ha documentado que la inmunosupresión sea un factor de riesgo en la infección por MERS-CoV (29). Tampoco se ha establecido una relación clara entre desenlaces adversos en COVID-19 e inmunosupresión (por cualquier causa incluida cáncer), y en los casos de mortalidad que se han documentado en pacientes inmunosuprimidos parecen coexistir otros factores de riesgo claros como enfermedad cardiovascular, edad avanzada, obesidad y sexo masculino (24). La información disponible hasta ahora no parece sugerir que los pacientes receptores de trasplante hepático tengan un mayor riesgo de enfermedad pulmonar grave al compararlos con la población general.

En pacientes receptores de trasplante hepático, la Sociedad Europea de Enfermedad Hepática sugiere que se deben usar alternativas como la telemedicina y la toma de pruebas analíticas en casa o en centros cercanos al paciente para minimizar exposición, y que no se recomienda modificar los esquemas de inmunosupresión para reducir el riesgo de infección por SARS-CoV-2 o complicaciones asociadas (25). Es importante tener en cuenta las interacciones entre inmunosupresores y los fármacos utilizados en el tratamiento de COVID-19, con una monitorización frecuente de los niveles de inmunosupresores (26). El uso concomitante de lopinavir/ritonavir con everolimus y sirolimus está contraindicado (25). En este grupo de pacientes también es importante mantener al día los esquemas de vacunación, según la edad y la comorbilidad del paciente $(25,26)$.

\section{COVID-19 Y PROGRAMAS DE TRASPLANTE HEPÁTICO}

Los centros de trasplante deben tener en cuenta que la realización de un trasplante hepático implica el uso de recursos como camas en la unidad de cuidado intensivo (UCI), ventiladores mecánicos, fármacos y derivados sanguíneos que podrían escasear durante la actual pandemia por COVID-19. También es importante tener en cuenta el riesgo de transmisión nosocomial del SARS-CoV-2 y el riesgo de contagio en pacientes hospitalizados por cualquier causa distinta a COVID-19. Cada centro de trasplante debe tener en cuenta estas consideraciones, al igual que las recomendaciones de las entidades gubernamentales locales, para decidir si continúan o no realizando trasplante hepático (26). En caso de que la decisión sea continuar con el programa de trasplante hepático de manera activa durante la pandemia, se recomienda priorizar únicamente a los pacientes más enfermos con un puntaje elevado en la escala de enfermedad hepática en estadio terminal (MELD, por sus siglas en inglés), falla hepática aguda sobre crónica o insuficiencia hepática aguda (25). La recomendación de la Asociación Americana de Estudio de Enfermedades Hepáticas es realizar pruebas para COVID-19 en los receptores potenciales que tengan síntomas que sugieran contagio, y en todos los donantes potenciales (26). Las alternativas son las pruebas por reacción en cadena de polimerasa y pruebas cualitativas o cuantitativas de inmunoglobulina (Ig) G y M. Una prueba molecular o una prueba cualitativa o cuantitativa para detección de IgM positiva en un potencial donante o receptor constituyen una contraindicación absoluta para realizar el trasplante.

\section{CONCLUSIONES}

Durante la actual pandemia de COVID-19 se ha documentado que los pacientes con enfermedad grave presentan una elevación de las transaminasas, trombocitopenia e hipoalbuminemia. Estas alteraciones pueden estar relacionadas con hepatitis viral debido a la respuesta inmunológica e inflamatoria excesiva y desproporcionada o por choque séptico, o inclusive por una enfermedad hepática crónica subyacente diagnosticada o no. Teniendo en cuenta la información disponible, estas alteraciones son marcadores de mal pronóstico y de progresión de la enfermedad. Pareciera que el daño hepático en sí mismo no contribuye a la causa de mortalidad en estos pacientes. Es importante tener en cuenta el diagnóstico diferencial de daño hepático inducido por medicamentos, y tomar precauciones para minimizar la toxicidad farmacológica en pacientes con enfermedad hepática establecida y COVID-19.

En los pacientes con enfermedad hepática crónica, cirrosis y en los receptores de trasplante hepático consideramos más prudente extremar las medidas para prevenir el contagio, utilizar alternativas de telemedicina y minimizar el contacto con otras personas y los traslados al hospital o centros de atención médica en la medida de lo posible. Las evaluaciones pretrasplante hepático en pacientes con enfermedad relativamente estable deben diferirse, y deben incluirse en lista de espera para trasplante hepático únicamente a los pacientes gravemente enfermos. Para los pacientes con carcinoma hepatocelular es importante continuar con las terapias locales y sistémicas siempre que sea posible, para evitar la progresión de la enfermedad. No existe evidencia clara que demuestre que hay peores desenlaces en este grupo de pacientes, pero sí coexisten los factores de riesgo tradicionales de mal pronóstico en COVID-19 con la enfermedad hepática o en la inmunosupresión por trasplante hepático; 
los desenlaces adversos parecen ser iguales que en la población sin patología hepática.

Con relación al manejo de la inmunosupresión, las recomendaciones para disminuir las dosis son muy específicas considerando linfopenia, fiebre persistente o progresión de la neumonía como argumentos para disminuir las dosis de antimetabolitos e inhibidores de la calcineurina. También se recomienda disminuir la dosis de esteroide sistémico en pacientes que reciben dosis altas de prednisona o equivalentes.

Es poca la información disponible específicamente para la población con hepatopatía crónica o antecedente de trasplante de hígado, probablemente por un subregistro de estos antecedentes en los estudios clínicos realizados. Extrapolamos muchos datos de la población asiática, pero como limitante hay que tener en cuenta que las características de la población son distintas en términos de edad y población de pacientes con hepatitis virales crónicas, cirrosis y trasplante hepático.

\section{REFERENCIAS}

1. WHO [Internet]. WHO; 2020 [actualización 11 de marzo de 2020; acceso 24 de abril de 2020]. Director-General's opening remarks at the media briefing on COVID-19 - 11 March 2020. Disponible en: https://www.who.int/dg/ speeches/detail/who-director-general-s-opening-remarksat-the-media-briefing-on-covid-19---11-march-2020

2. European Center for Disease Prevention and Control [Internet]. ECDC; 2019. [actualización 2 de abril de 2020; acceso 24 de abril de 2020]. Situation update worldwide, as of 02 April 2020. Disponible en: https://www.ecdc.europa. eu/en/geographical-distribution-2019-ncov-cases

3. Instituto Nacional de Salud [Internet]. INS; 2020 [acceso 24 de abril de 2020]. COVID-19 Colombia - Reporte 01-04-2020-Corte 1pm. Disponible en: https://www.ins. gov.co/Noticias/Paginas/Coronavirus.aspx

4. Chau TN, Lee KC, Yao H, et al. SARS-associated viral hepatitis caused by a novel coronavirus: report of three cases. Hepatology. 2004; 39:302-10. https://doi.org/10.1002/ hep. 20111

5. Alsaad KO, Hajeer AH, Al Balwi M, et al. Histopathology of Middle East respiratory syndrome coronavirus (MERS$\mathrm{CoV}$ ) infection - Clinicopathological and ultrastructural study. Histopathology. 2018; 78:516-24. https://doi. org/10.1111/his.13379

6. Xu L, Liu J, Lu M, Yang D, Zheng X. Liver injury during highly pathogenic human coronavirus infections. 2020. https: / / doi.org/10.1111/liv.14433

7. Li W, moor MJ, Vasilieva N, et al. Angiotensin-converting enzime 2 is a functional receptor for the SARS coronavirus. Nature. 2003;426(6965):450-54. https://doi. org/10.1038/nature02145

8. Hamming I, Timens W, Bulthuis $\mathrm{M}$, et al. Tissude distribution of ACE2 protein, the functional receptor for SARS coronavirus. A first step in understanding SARS pathogene- sis. J Patho. 2004;203(2):631-37. https://doi.org/10.1002/ path. 1570

9. Tan YJ, Fielding BC, Goh PY, et al. Overexpression of 7a, a protein specifically encoded by thesever acute respiratory syndrome coronavirus, induces apoptosis via a caspasedepedent pathway. J Virol. 2004;78(24):14043-47. https:// doi.org/10.1128/JVI.78.24.14043-14047.2004

10. Lu J, Zhao J, Li N, et al. Ultrastructurte pathology of all organs in sever acute respiratory syndrome. Chinese Journal of Diagnostic Pathology. 2003;04:72-7.

11. Saad M, Omrani AS, Baig K, et al. Clinical aspects and outcomes of 70 patients with Middle East respiratory syndrome coronavirus infection: a single-center experience in Saudi Arabia. Int J Infect Dis. 2014;29:301-06. https:// doi. org/10.1016/j.ijid.2014.09.003

12. Arabi YM, Arifi AA, Balkhy $\mathrm{HH}$, et al. Clinical course and outcomes of critically ill patients with Middle East respiratory syndrome coronavirus infection. Ann Intern Med. 2014:160(6):389-97. https://doi.org/10.7326/M13-2486

13. Zhang C, Shi L, Wang FS. Liver injury in COVID-19: management and challenges. Lancet Gastroenterol Hepatol. 2020. https://doi.org/10.1016/S2468-1253(20)30057-1

14. Chen N, Zhou M, Qu J, Gong F, Han Y, Qiu Y, et al. Epidemiological and clinical characteristics of 99 cases of 2019 novel coronavirus pneumonia in Wuhan, China: a descriptive study. Lancet. 2020; 395:507-13. https://doi. org/10.1016/S0140-6736(20)30211-7

15. Wu C, Chen Z, Cai Y, Xia J, Zhou X, Xu S, et al. Risk factors associated with acute respiratory distress syndrome and death in patients with coronavirus disease 2019 Pneumonia in Wuhan, China. JAMA Intern Med. https// doi.org/10.1001/jamainternmed.2020.0994

16. Zhou F, Yu T, Du R, Fan G, Liu Y, Liu Z, et al. Clinical course and risk factors for mortality of adult inpatients with COVID-19 in Wuhan, China: a retrospective cohort study. Lancet. 2020;395:1054-62. https://doi.org/10.1016/ S0140-6736(20)30566-3

17. Wang D, Hu B, Zhu F, Liu X, Zhang J, Wang B, et al. Clinical characteristics of 138 patients with 2019 novel coronavirus-infected pneumonia in Wuhan-China. JAMA. 2020. https://doi.org/10.10001/jama.2020.1585.

18. Rismanbaf A, Zarei S. Liver and Kidney Injuries in COVID19 and Their Effects on DrugTherapy; a Letter to Editor. Archives of Academic Emergency Medicine. 2020;8(1):e17.

19. Yang Z, Xu M, Yi JQ et al. Clinical characteristics and mechanism of liver damage in patients with severe acute respiratory syndrome. Hepatobiliary Pancreat Dis Int. 2005;4(1):60-63.

20. Bangash MN, Patel J, Parekh D. COVID-19 and the liver: little cause for concern. Lancet Gastroenterol Hepatol. 2020. https://doi.org/10.1016/S2468-1253(20)30084-4

21. Zhang B, Zhou X, Qiu Y, et al. Clinical characteristics of 82 death cases with COVID-19. medRxiv. 2020. https://doi. org/10.1101/2020.02.26.20028191

22. Huang Y, Gao Z. Study of the relationship SARS and hepatitis virus B. Chin J Clini Hepatol. 2003;6:342-343. 
23. Mehta P, McAuley DF, Brown M, Sanchez E, Tattersall RS, Manson JJ. COVID-19: consider cytokine storm syndromes and immunosuppression. The Lancet. 2020. https:// doi.org/10.1016/S0140-6736(20)30628-0

24. Xiao Y, Pan H, She Q, Wang F, Chen M. Prevention of SARS-CoV-2 infection in patients with decompensated cirrhosis. Lancet Gastroenterol Hepatol. 2020. https://doi. org/10.1016/S2468-1253(20)30080-7

25. Boettler, T. Newsome PN, Mondelli MU, Maticic M, Cordero E, Cornberg M, Berg T. Care of patients with liver disease during the COVID-19 pandemic: EASL-ESCMID Position Paper. JHEP Reports. https://doi.org/10.1016/j. jhepr.2020.100113

26. American Association For The Study of Liver Disease [Internet]. AASLD; 2020 [actualización 16 de abril de 2020; acceso 24 de abril de 2020]. Clinical insights for hepatology and liver transplant providers during the
COVID-19 Pandemic. Disponible en: https://www.aasld. org/sites/default/files/2020-03/AASLD-COVID 19ClinicalInsights-3.23.2020-FINAL-v2.pdf

27. D’Antiga L. Coronaviruses and immunosuppressed patients. The facts during the thrid epidemic. Liver Transpl. 2020. https://doi.org/10.1002/lt.25756

28. Guo YR, Cao QD, Hong ZS, Tan YY, Chen SD, Jin HJ, et al. The origin, transmission and clinical therapies on coronavirus disease 2019 (COVID-19) outbreak - an update on the status. Military Medical Research. 2020;7(1):11. https:// doi.org/10.1186/s40779-020-00240-0

29. Kumar D, Tellier R, Draker R, Levy G, Humar A. Severe Acute Respiratory Syndrome (SARS) in a Transplant Recipient and Guidelines for Donor SARS Screening. American Journal of Transplantation. 2003;3(8):977-81. https://doi.org/10.1034/j.1600-6143.2003.00197.x 